\title{
Quality of Preventive Care for Diabetes: Effects of Visit Frequency and Competing Demands
}

\author{
Josbua J. Fenton, MD, MPH \\ Michael Von Korff, ScD ${ }^{2}$ \\ Elizabeth H.B. Lin, MD, MPH \\ Paul Ciechanowski, MD, $M P H^{3}$ \\ Bessie A. Young, MD, MPH \\ 'Department of Family and Community \\ Medicine, University of California, Davis, \\ Sacramento, Calif \\ ${ }^{2}$ Center for Health Studies, Group Health \\ Cooperative, Seattle, Wash \\ ${ }^{3}$ Department of Psychiatry and Behavioral \\ Sciences, University of Washington, \\ Seattle, Wash \\ ${ }^{4}$ Department of Medicine, University of \\ Washington, and Veterans Administration \\ Hospital, Seattle, Wash
}

\begin{abstract}
PURPOSE We sought to determine the association between timely receipt of diabetes-related preventive services and the longitudinal pattern of outpatient service use as characterized by a novel taxonomy that prioritized visits based on the Oregon State Prioritized Health Services List.
\end{abstract}

METHODS We performed a cross-sectional analysis of mail survey and automated health care data for a population-based sample of patients with diabetes enrolled in a health maintenance organization in Washington State $(N=4,463)$. Outcomes included American Diabetes Association-recommended preventive services, including regular hemoglobin $\mathrm{A}_{1 \mathrm{c}}\left(\mathrm{HbA}_{1 \mathrm{c}}\right)$ monitoring, retinal examination, and microalbuminuria screening. Patients with fewer than 8 visits during the 2-year study period were considered infrequent users, while patients with 8 or more visits were classified as lower-priority users if most visits were for conditions of relatively low rank on the Oregon list and as higher-priority users otherwise.

RESULTS After adjustment for social, demographic, and clinical factors, and depression, infrequent users had significantly reduced odds of receiving at least $1 \mathrm{HbA}_{1 \mathrm{c}}$ test (odds ratio $[\mathrm{OR}]=0.35,95 \%$ confidence interval $[\mathrm{Cl}], 0.24-0.51)$, retinal examination $(\mathrm{OR}=0.74,95 \% \mathrm{Cl}, 0.63-0.86)$, and microalbuminuria screening (OR $=0.75,95 \% \mathrm{Cl}, 0.58-0.96)$ relative to higher-priority users during the previous year. Lower-priority users also had relatively reduced odds of receiving at least 1 $\mathrm{HbA}_{1 \mathrm{c}}$ test $(\mathrm{OR}=0.59,95 \% \mathrm{Cl}, 0.35-1.01)$, retinal examination $(\mathrm{OR}=0.68,95 \%$ $\mathrm{Cl}, 0.56-0.84)$, and microalbuminuria screening $(\mathrm{OR}=0.79,95 \% \mathrm{Cl}, 0.57-1.09)$ despite attending a similar mean number of total visits as higher-priority users.

CONCLUSIONS Patients who attend relatively few outpatient visits or who attend more frequent visits for predominantly lower-priority conditions are more likely to receive substandard preventive care for diabetes.

Ann Fam Med 2006:4:32-39. DOI: 10.1370/afm.421.

\section{INTRODUCTION}

$\mathrm{R}$ egular medical care can prevent many common diabetes complications, including ischemic heart disease, stroke, retinopathy, nephropathy, and neuropathy. ${ }^{1}$ The American Diabetes Association (ADA) recommends that persons with diabetes receive at least semiannual hemoglobin $\mathrm{A}_{1 \mathrm{C}}\left(\mathrm{HbA}_{1 \mathrm{C}}\right)$ monitoring, annual retinal examination to monitor or screen for retinopathy, and annual microalbuminuria testing to screen for nephropathy. ${ }^{2}$ The delivery of ADA-recommended services has been estimated to require 2 to 4 annual medical visits for most patients with diabetes. ${ }^{2}$

Despite the effectiveness of preventive care for diabetes, many patients do not receive recommended services. ${ }^{3}$ One contributing factor may be that some patients simply do not make regular clinic visits for diabetes care, and patients who receive infrequent outpatient monitoring may be 
less likely to receive recommended preventive services. ${ }^{4,5}$ Additionally, many patients with diabetes have comorbid chronic illness, and the exigencies of managing comorbid conditions may distract clinicians from the delivery of recommended preventive services. ${ }^{6-8}$ Moreover, acute illnesses account for the majority of primary care visits, and clinicians are much less likely to perform tasks that may facilitate prevention during acute illness visits. ${ }^{9}$ Thus, longitudinally, both the quantity and content of outpatient care may affect the delivery of timely preventive services for diabetes.

In this study, we used a novel taxonomy of outpatient visits to characterize the pattern of outpatient service use among a population-based sample of patients with diabetes. The taxonomy classified patients by the quantity of outpatient visits over a 2-year period, the types of services received (eg, acute vs chronic illness), and the relative effectiveness of the services received, with more effective services designated higher priority and less effective services, lower priority. From the perspective of the health system, we then framed 2 hypotheses related to the delivery of diabetes-related preventive services. First, we hypothesized that patients who infrequently attended outpatient visits would be at increased risk for deferred diabetes-related preventive services, because clinicians may have insufficient opportunity to recommend preventive services to these patients. Second, we hypothesized that patients with diabetes who frequently sought care for lower-priority conditions would be less likely to receive timely diabetes-related preventive services, because patient demand for relatively lower-priority services may compete for clinicians' time and attention during office visits, thereby reducing the likelihood of preventive service delivery. We focused on the competing demand for lower-priority medical care, because health systems would ideally devote limited resources to care of proven effectiveness with the greatest potential societal health benefit. If many patients receive care for lower-priority conditions instead of recommended preventive services, interventions may be needed to ensure the delivery of effective preventive services among many patients who may preferentially seek care for such conditions.

\section{METHODS}

\section{Design, Setting, and Patients}

We performed a population-based, cross-sectional study of adult enrollees with diabetes within Group Health Cooperative (GHC), a staff-model health maintenance organization serving approximately 450,000 persons in western Washington State. The methods of sampling have been previously described.$^{10}$ In 2001, a mail survey was conducted to estimate the prevalence of depression among adult patients with diabetes who were identified from a validated centralized diabetes registry that includes enrollees based on antidiabetic drug prescriptions, laboratory abnormalities, and outpatient and inpatient diagnostic codes. ${ }^{11}$ The survey response rate was $61.7 \%$, including 4,463 subjects who responded to a depression screen and provided informed consent for linkage to automated clinical and pharmacy data. Within GHC, diabetes care is generally provided by office-based primary care clinicians. Although clinicians have access to electronic diabetes flow sheets populated with registry data, no general policies dictate how individual practices use the registry, and there are no formal systems in place to remind patients that diabetes preventive services are due. The institutional review boards of GHC and the University of Washington approved the study protocol.

\section{Patterns of Outpatient Use}

We characterized patients' pattern of outpatient service use with a newly developed taxonomy of outpatient visits that relies on International Classification of Diseases, Ninth Revision (ICD-9) diagnostic codes, which GHC clinicians identify after outpatient encounters. To develop the taxonomy, we categorized each ICD-9 code resulting from an adult outpatient visit in 2002 into 1 of 7 major diagnostic categories: acute diseases, chronic diseases, symptoms and ill-defined conditions, mental illnesses, vision and hearing disorders, dermatologic diseases, and preventive care and pregnancyrelated conditions. We defined chronic diseases as conditions that typically last for more than 3 months, are recurrent, or have a chronic course in one quarter or more of incident cases. Symptoms and ill-defined conditions included most ICD-9 diagnoses from codes 780 to 799 but also some chronic symptomatic conditions that have uncertain pathophysiology or lack objective diagnostic signs or therapies of proven effectiveness (eg, irritable bowel syndrome).

We subsequently used the Oregon State Prioritized Health Services List ${ }^{12}$ to subclassify acute and chronic diseases into higher-priority diseases (those for which medical care provides proven or likely benefit) and lower-priority diseases (those for which medical care provides lesser benefit in terms of morbidity, mortality, or quality of life). Using an evidence-based methodology, commissioners for the Oregon Health Plan created the prioritized list of more than 700 diagnosis and treatment pairs to assist in defining the benefits of Medicaid expansion. ${ }^{13}$ To prioritize acute and chronic diseases, we mapped ICD-9 diagnoses in these diagnostic categories to corresponding diagnoses on the prioritized list. If the diagnosis ranked 350 or greater on the list, we classified the disease as higher prior- 
ity; otherwise, we preliminarily classified the disease as lower priority. Three coauthors (JJF, MVK, EHL) independently reviewed each diagnosis that mapped to diseases of intermediate rank on the Oregon list (codes 351-549) and decided by consensus to reclassify a substantial number of diseases as higher priority (eg, acute sinusitis). To assess coding reliability, another clinician (blinded to the initial classification results) recoded the diagnostic category of the 279 most common acute, chronic, or ill-defined condition diagnoses, and the priority of the 142 most common diagnoses that fell in the intermediate-priority range on the Oregon list. The clinician's coding of diagnostic category and priority agreed with that of the 3 -coauthor committee for $81.4 \%$ and $80.3 \%$ of diagnoses, respectively, and agreement for both was $75.3 \%$.

We used automated health care data to identify all outpatient visits made by patients during the 2-year period surrounding their survey dates. We categorized each visit by its major diagnostic category using its associated ICD-9 codes. When a visit had more than 1 ICD-9 code, we weighted each by the reciprocal of the total number of codes. We then generated counts of outpatient visits within each diagnostic category for patients during the study period.

Our goal was to identify patients who used relatively few outpatient services and, among more frequent users, to distinguish patients seeking care for predominantly higher-priority acute and chronic diseases from those who received a preponderance of care for lower-priority acute and chronic diseases, and symptomatic conditions. We therefore categorized patients based on their overall frequency of acute, chronic, and symptomatic illness visits and the relative priority of conditions addressed during these visits. We excluded visits for mental illnesses, vision and hearing disorders, and preventive care and pregnancy-related conditions because they were less relevant to characterizing patients' care for acute and chronic illness. We also excluded visits for dermatologic conditions, because the most common diagnostic codes within this category were nonspecific and difficult to prioritize. When a visit included diagnoses within excluded categories along with acute, chronic, and symptomatic illness diagnoses, we counted only the visit fraction associated with the latter diagnoses.

After examining the distribution of acute, chronic, and symptomatic visits, we set a threshold for infrequent visits of these types of 8 in a 2 -year period. We classified patients with fewer than 8 visits as infrequent users. Among patients with 8 or more visits, we classified patients as lower-priority users if one half or more of their visits were for lower-priority diagnoses or ill-defined conditions, and as higher-priority users if less than one half of their visits were for lower-priority diagnoses or ill-defined conditions.

\section{Use of Diabetes-Related Preventive Services}

We used automated administrative and laboratory data for patients during the 2 years before survey completion to measure the receipt of ADA-recommended preventive services. ${ }^{2}$ We examined 2 outcomes related to monitoring for glycemic control in the previous year: (1) receipt of at least $1 \mathrm{HbA}_{1 \mathrm{C}}$ test and (2) receipt of at least $3 \mathrm{HbA}_{1 \mathrm{C}}$ tests among patients with poor glycemic control (ie, those whose most recent $\mathrm{HbA}_{1 \mathrm{C}}$ value was $\geq 8.0 \%$ ). Two outcomes reflected timely monitoring for diabetic retinopathy: (1) at least 1 screening retinal examination in the previous year and (2) at least 2 retinal examinations in the 2 previous years among patients known to have retinopathy (ie, annual surveillance). Finally, among patients who had not been prescribed an angiotensin-converting enzyme (ACE) inhibitor before the survey $(41.9 \%)$, we determined whether each received microalbuminuria screening during the previous year.

\section{Social, Demographic, and Clinical Covariates}

The mail survey elicited information for social and demographic covariates (education, marital status, and race) and height and weight (to compute body mass index). Each patient also completed the Patient Health Questionnaire-9, a validated screening instrument that provides dichotomous diagnoses of major and minor depression. ${ }^{14}$

We used automated diagnostic data to identify 7 complications of diabetes (retinopathy, nephropathy, neuropathy, cerebrovascular disease, cardiovascular disease, peripheral vascular disease, and ketoacidosis) ${ }^{15}$ and automated laboratory data to obtain the $\mathrm{HbA}_{1 \mathrm{C}}$ result nearest the survey date. We used pharmacy data to identify prescriptions for oral hypoglycemic medications and insulin (as an indicator of treatment intensity) and to compute an index of chronic disease comorbidity (an RxRisk score), which is as predictive of future health care costs as ambulatory care groups. ${ }^{16} \mathrm{We}$ excluded medications for diabetes and depression when computing the index to avert duplicate measurement of these diagnoses.

\section{Analyses}

We performed bivariate comparisons of characteristics of infrequent, lower-priority, and higher-priority users, followed by $\chi^{2}$ tests to determine the association between use pattern and preventive service outcomes. We then used logistic regression analysis to test the hypothesis that infrequent and lower-priority users would be less likely to receive timely preventive ser- 
vices after adjusting for potentially confounding factors identified in bivariate analyses. The reference group in these models was higher-priority users, and each model included age, sex, marital status, education, chronic disease comorbidity (quartile of RxRisk scores), number of diabetes complications, treatment intensity, depression status (none, minor, and major), and primary care clinic. We selected these covariates because each was significantly associated with use pattern in bivariate analyses and would plausibly affect delivery of preventive services. In subsequent adjusted analyses, we found that smoking and body mass index had no substantive effect on study results and so did not include them in final models.

Analyses of survey nonresponse bias have been previously described in detail. ${ }^{10}$ In brief, nonrespondents were younger, were more likely to be using insulin, and had higher $\mathrm{HbA}_{1 \mathrm{C}}$ values, and had a higher prevalence of heart disease. To judge whether nonresponse bias affected our results, we repeated the analyses with observations weighted by the inverse of the predicted probability of response (propensity score). ${ }^{17}$ Because there were only trivial differences in weighted and unweighted analyses, we report the unweighted analyses here. We used 2-sided hypothesis tests and an $\alpha$ of .05.

\section{RESULTS}

\section{Sample Characteristics}

The patients had a mean age of 65 years and a broad range of diabetes treatment intensities and complications (Table 1). About 1 in 5 patients were nonwhite,

\section{Table 1. Social, Demographic, and Clinical Characteristics of Patients by Pattern of Use} of Outpatient Services

\begin{tabular}{|c|c|c|c|c|}
\hline \multirow[b]{2}{*}{ Characteristic $^{\dagger}$} & \multirow[b]{2}{*}{$\begin{array}{l}\text { Total Sample } \\
(\mathrm{N}=4,463)\end{array}$} & \multicolumn{3}{|c|}{ Pattern of Use* } \\
\hline & & $\begin{array}{c}\text { Infrequent } \\
(n=1,576)\end{array}$ & $\begin{array}{c}\text { Lower-Priority } \\
(n=542)\end{array}$ & $\begin{array}{l}\text { Higher-Priority } \\
(n=2,345)\end{array}$ \\
\hline Women, No. (\%) & $2,175(48.7)$ & $663(42.1)$ & $321(59.2)$ & $1,191(50.1)$ \\
\hline High school education or less, No. (\%) & $1,088(24.7)$ & $345(22.1)$ & $115(21.4)$ & $628(27.1)$ \\
\hline \multicolumn{5}{|l|}{ Marital status, No. (\%) } \\
\hline Single & $431(10.0)$ & $177(11.3)$ & $48(8.9)$ & $206(8.9)$ \\
\hline Married/living as & $2,925(66.0)$ & $1,077(68.8)$ & $348(64.6)$ & $1,500(64.5)$ \\
\hline Widowed & $547(12.4)$ & $132(8.4)$ & $72(13.4)$ & $343(14.8)$ \\
\hline Divorced or separated & $526(11.8)$ & $179(11.4)$ & $71(13.2)$ & $276(11.9)$ \\
\hline Nonwhite race, No. (\%) & $892(20.4)$ & $334(37.4)$ & $111(12.4)$ & $447(50.1)$ \\
\hline Age, y, mean (SD) & $64.9(12.6)$ & $60.1(12.7)$ & $61.5(13.7)$ & $65.8(13.2)$ \\
\hline \multicolumn{5}{|l|}{ RxRisk comorbidity score, No. (\%) } \\
\hline$<1,300$ & $915(20.5)$ & $527(33.4)$ & $118(21.8)$ & $270(11.5)$ \\
\hline $1,300-2,599$ & $1,147(25.7)$ & $506(32.1)$ & $145(26.8)$ & $496(21.2)$ \\
\hline $2,600-4,399$ & $1,178(26.4)$ & $387(24.6)$ & $157(29.0)$ & $634(27.0)$ \\
\hline$\geq 4,400$ & $1,223(27.4)$ & $156(9.9)$ & $122(22.5)$ & $945(40.3)$ \\
\hline \multicolumn{5}{|l|}{ Diabetes complications, No. (\%) } \\
\hline 0 & $1,404(31.4)$ & $774(49.1)$ & $183(33.8)$ & $447(19.1)$ \\
\hline 1 & $1,405(31.5)$ & $549(34.8)$ & $199(36.7)$ & $657(28.0)$ \\
\hline 2 & $853(19.1)$ & $187(11.9)$ & $112(20.7)$ & $554(23.6)$ \\
\hline$\geq 3$ & $801(17.9)$ & $66(4.2)$ & $48(8.9)$ & $687(29.3)$ \\
\hline $\mathrm{HbA}_{1 \mathrm{c}} \geq 8.0 \%$, No. (\%) & $1,982(46.3)$ & $682(46.5)$ & $197(38.0)$ & $1,103(48.2)$ \\
\hline \multicolumn{5}{|l|}{ Treatment intensity, No. (\%) } \\
\hline None or diet & $1,134(25.4)$ & $462(29.3)$ & $188(34.7)$ & $484(20.6)$ \\
\hline Oral hypoglycemic agent & $1,986(44.5)$ & $790(50.1)$ & $252(46.5)$ & $944(40.3)$ \\
\hline Insulin \pm oral hypoglycemic & $1,343(30.1)$ & $324(20.6)$ & $102(18.8)$ & $917(39.1)$ \\
\hline $\mathrm{BMI} \geq 30 \mathrm{~kg} / \mathrm{m}^{2}$, No. $(\%)$ & $2,147(48.8)$ & $727(46.8)$ & $308(58.0)$ & $1,112(48.2)$ \\
\hline Smoking currently, No. (\%) & $381(8.6)$ & $184(11.8)$ & $44(8.3)$ & $153(6.7)$ \\
\hline \multicolumn{5}{|l|}{ Depression status, No. (\%) } \\
\hline Not depressed & $3,552(79.5)$ & $1,344(85.3)$ & $410(75.7)$ & $1,798(76.7)$ \\
\hline Minor depression & $375(8.4)$ & $108(6.9)$ & $47(8.7)$ & $220(9.4)$ \\
\hline Major depression & $536(12.0)$ & $124(7.9)$ & $85(15.7)$ & $327(13.9)$ \\
\hline
\end{tabular}




\begin{tabular}{|c|c|c|c|c|}
\hline \multirow[b]{2}{*}{ Type of Visit* } & \multirow[b]{2}{*}{$\begin{array}{l}\text { Total Sample } \\
(\mathrm{N}=4,463)\end{array}$} & \multicolumn{3}{|c|}{ Pattern of Use } \\
\hline & & $\begin{array}{c}\text { Infrequent } \\
(\mathrm{n}=1,576)\end{array}$ & $\begin{array}{c}\text { Lower-Priority } \\
(\mathrm{n}=542)\end{array}$ & $\begin{array}{c}\text { Higher-Priority } \\
(\mathrm{n}=2,345)\end{array}$ \\
\hline Acute disease-higher priority & $1.9(2.7)$ & $0.5(0.8)$ & $1.9(1.9)$ & $2.8(3.2)$ \\
\hline Acute disease-lower priority & $1.3(1.8)$ & $0.5(0.8)$ & $3.2(2.7)$ & $1.4(1.6)$ \\
\hline Chronic disease-higher priority & $7.5(7.4)$ & $3.0(1.6)$ & $4.8(3.1)$ & $11.2(8.4)$ \\
\hline Chronic disease-lower priority & $1.3(1.9)$ & $0.3(0.6)$ & $3.1(2.8)$ & $1.5(1.9)$ \\
\hline Symptoms and ill-defined conditions & $1.6(2.4)$ & $0.4(0.7)$ & $4.2(4.2)$ & $1.8(2.0)$ \\
\hline Total & $13.6(11.2)$ & $4.8(2.0)$ & $17.3(9.4)$ & 18.7 (11.5) \\
\hline
\end{tabular}

* Values are mean (SD) numbers of visits. Excludes visits for dermatologic diagnoses, vision and hearing, mental health illness, and preventive and pregnancy-related services. All comparisons across use patterns are statistically significant $(P<.001)$.

and $12 \%$ had major depression. More than one third (35\%) were infrequent users, whereas 1 in $8(12 \%)$ were lower-priority users and more than one half (52\%) were higher-priority users. Infrequent users were younger and predominantly male, and they had less chronic disease comorbidity, fewer diabetes complications, and a lower prevalence of depression than lower- or higher-priority users (Table 1 ). Lower-priority users were predominantly female, were more likely to be treated by diet alone, and had intermediate levels of chronic disease and a higher prevalence of major depression. Higher-priority users tended to be older and to have less education and higher rates of diabetes complications and chronic disease comorbidity.
Lower- and higher-priority users had a similar mean number of visits during the study period however, $^{-}$ higher-priority users made more than twice as many visits for higher-priority chronic diseases (Table 2). During the 2-year study period, infrequent users had a mean of fewer than 5 visits, of which approximately 3 were for higher-priority chronic diseases.

\section{Receipt of Preventive Services}

Higher-priority users consistently had the highest rates of timely preventive services for diabetes, whereas lower-priority users typically had rates that were intermediate between those of higher-priority and infrequent users (Figure 1). Among patients with poorly

\section{Figure 1. Receipt of diabetes preventive services by patterns of use.}

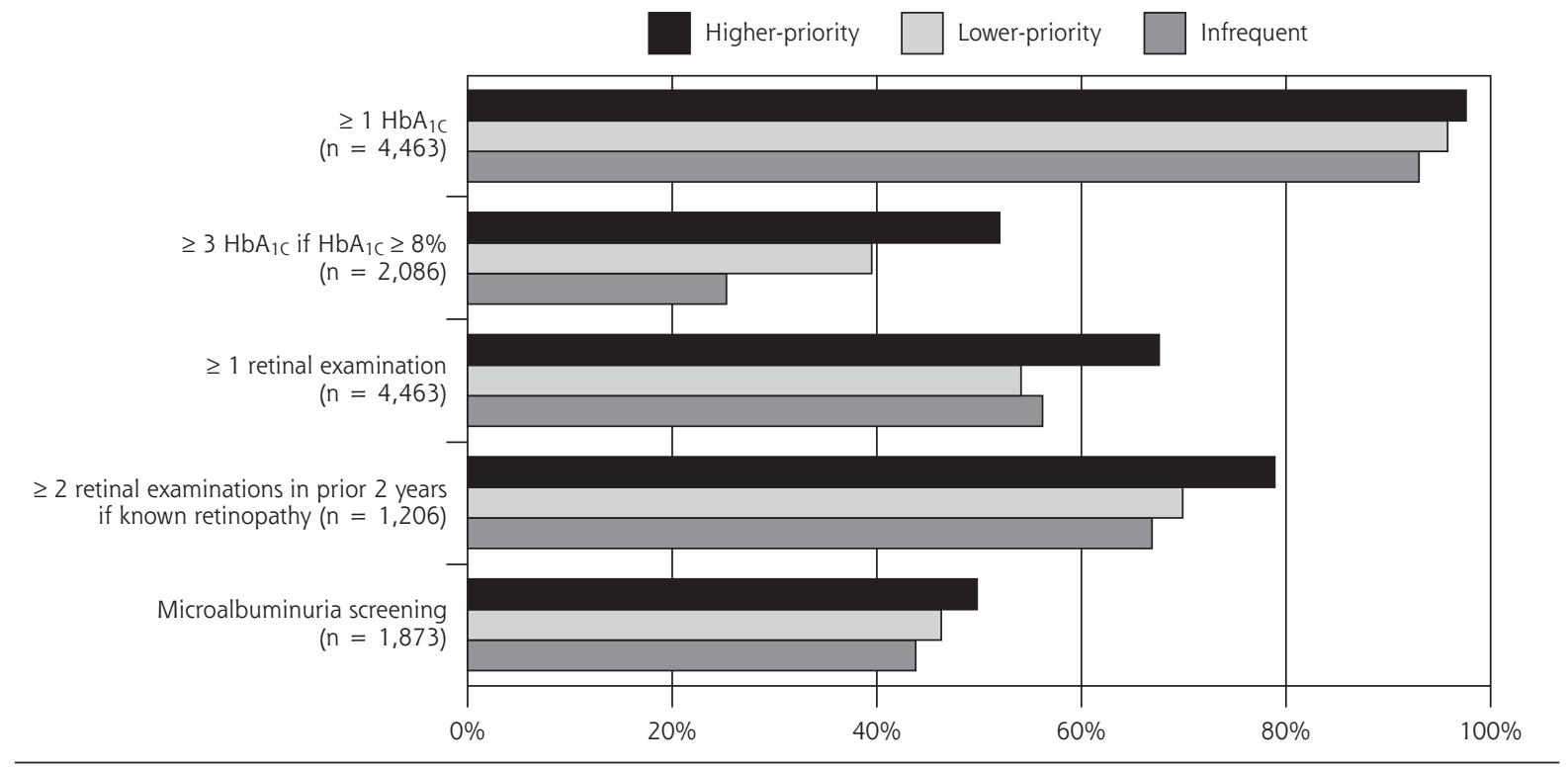

$\mathrm{HbA}_{1 \mathrm{C}}=$ hemoblogin $\mathrm{A}_{1 \mathrm{c}}$.

Note: Values are rates of receipt of services over a 2-year period. Microalbuminuria screening was assessed only in patients who did not have a prescription for an angiotension-converting enzyme inhibitor at baseline. Comparison of outcomes across use patterns are statistically significant $(P<.001)$, except for the comparison of microalbuminuria screening $(P=.05)$ 
controlled diabetes $\left(\mathrm{HbA}_{1 \mathrm{C}}\right.$ values $\left.\geq 8.0 \%\right)$, the majority $(54.9 \%)$ of higher-priority users had received 3 or more $\mathrm{HbA}_{1 \mathrm{C}}$ tests in the previous year, whereas rates of regular $\mathrm{HbA}_{1 \mathrm{C}}$ monitoring were significantly lower among lower-priority users $(44.4 \%)$ and infrequent users $(29.5 \%)(P<.001)$.

With regard to screening for diabetic retinopathy, higher-priority users had a significantly higher rate of timely screening during the previous year $(67.6 \%)$, whereas the screening rate was comparable among lower-priority users $(54.1 \%)$ and infrequent users $(56.2 \%)(P<.001)$. A similar pattern was seen among patients with known retinopathy, for whom annual surveillance examination is recommended.

Finally, higher-priority users were the most likely and infrequent users were the least likely to have received microalbuminuria screening during the previous year $(P=.05)$.

After adjustment for potentially confounding social, demographic, and clinical factors, a pattern of lower-priority or infrequent use remained associated with reduced odds of receiving timely $\mathrm{HbA}_{1 \mathrm{C}}$ monitoring, screening or surveillance retinal examinations, and microalbuminuria screening (Table 3 ). Relative to higher-priority users, infrequent users had significantly reduced odds across each preventive service outcome. Lower-priority users had significantly reduced odds of timely retinal screening or surveillance relative to higher-priority users and consistent but nonsignificant trends toward reduced odds of timely $\mathrm{HbA}_{1 \mathrm{C}}$ monitoring and microalbuminuria screening.

\section{DISCUSSION}

We found that patients who made infrequent visits were least likely to receive timely diabetes-related preventive care. We also found that patients who made frequent visits for lower-priority health conditions were at increased risk for delayed diabetes-related preventive services despite making a number of total visits comparable to that of a group that typically received care for higher-priority conditions. Our findings are therefore consistent with our hypotheses that patterns of infrequent or lower-priority outpatient use would be associated with lower-quality diabetes preventive care.

Infrequent users were at the highest relative risk for not receiving recommended preventive services. Similarly, patients with diabetes who had 2 or fewer annual visits to suburban Los Angeles primary care practices were less likely to receive timely proteinuria and lipid testing, although this analysis did not account for specialty clinic visits. ${ }^{4}$ Patients with diabetes treated by diet alone are less likely to receive timely $\mathrm{HbA}_{1 \mathrm{C}}$ testing, microalbuminuria testing, or retinal screening, ${ }^{5}$ which may be attributable to less frequent clinic visits among patients managed in this way. ${ }^{18}$ Infrequent users in our sample made a mean of 3 higher-priority chronic disease visits during the 2-year study, while lower-priority users made approximately 5. Currently, more than one third of US patients with diabetes visit their physicians less than quarterly. ${ }^{18}$ Given the complexity of diabetes management, it may be difficult to deliver high-quality diabetes care during 2 or 3 annual office encounters. Office systems that facilitate patient follow-up, opportunistic prevention, and guideline adherence might improve rates of diabetes preventive care among subpopulations of infrequent users, such as younger men with less comorbidity. ${ }^{11}$

Lower-priority users were frequent users of outpatient services but had a preponderance of visits for lower-priority or ill-defined conditions. During outpatient visits, physicians must resolve the tension between multiple competing demands, which may include the evaluation of patient complaints, chronic illness care, or preventive and counseling services. ${ }^{19}$ When patients have acute symptoms, primary care physicians are much

Table 3. Adjusted Odds of Timely Receipt of Diabetes-Related Preventive Services by Pattern of Use

\begin{tabular}{|c|c|c|c|}
\hline \multirow[b]{2}{*}{ Diabetes-Related Preventive Service } & \multicolumn{3}{|c|}{ Pattern of Use* } \\
\hline & $\begin{array}{l}\text { Infrequent } \\
\text { OR }(95 \% \mathrm{Cl})^{\dagger}\end{array}$ & $\begin{array}{l}\text { Lower-Priority } \\
\text { OR }(95 \% \mathrm{Cl})^{\dagger}\end{array}$ & $\begin{array}{l}\text { Higher-Priority } \\
\text { OR }(95 \% \mathrm{CI})^{\dagger}\end{array}$ \\
\hline$\geq 1 \mathrm{HbA}_{1 \mathrm{c}}$ test in previous year $(\mathrm{n}=4,347)$ & $0.35(0.24-0.51)$ & $0.59(0.35-1.01)$ & ref \\
\hline$\geq 3 \mathrm{HbA}_{1 \mathrm{c}}$ tests in previous year if $\mathrm{HbA}_{1 \mathrm{c}} \geq 8 \%(\mathrm{n}=1,837)$ & $0.44(0.35-0.56)$ & $0.80(0.57-1.12)$ & ref \\
\hline$\geq 1$ retinal examinations in previous year $(n=4,347)$ & $0.74(0.63-0.86)$ & $0.68(0.56-0.84)$ & ref \\
\hline $\begin{array}{l}\geq 2 \text { retinal examinations in } 2 \text { previous years if known retinopathy } \\
\quad(\mathrm{n}=1,179)\end{array}$ & $0.57(0.40-0.81)$ & $0.55(0.34-0.89)$ & ref \\
\hline$\geq 1$ microalbuminuria screenings in previous year $(n=1,831)^{\ddagger}$ & $0.75(0.58-0.96)$ & $0.79(0.57-1.09)$ & ref \\
\hline \multicolumn{4}{|c|}{$\begin{array}{l}\mathrm{OR}=\text { odds ratio; } \mathrm{Cl}=\text { confidence interval; } \mathrm{HbA}_{1 \mathrm{c}}=\text { hemoglobin } \mathrm{A}_{1 c} \text {; ref }=\text { reference group. } \\
\text { * Includes fewer patients than bivariate analyses because of missing data. } \\
\text { † Odds ratios adjusted for age, sex, marital status, ethnicity, education, comorbidity (RxRisk score), number of diabetes complications, treatment intensity, depression status, } \\
\text { and clinic site. } \\
\text { † Among patients not prescribed an angiotensin-converting enzyme inhibitor. }\end{array}$} \\
\hline
\end{tabular}


less likely deliver preventive or counseling services or to engage in activities that would facilitate receipt of preventive care. ${ }^{20,21}$ Lower-priority users may be less likely to receive preventive services if patient concerns about acute or chronic symptoms divert clinicians' attention from the delivery of preventive services for diabetes. Although studies suggest that competing demands affect depression management ${ }^{22}$ and cancer screening, ${ }^{7,8}$ we are unaware of previous research examining the impact of competing demands on diabetes care. Primary care clinicians should be aware that patients with diabetes who frequently seek care for lower-priority conditions are at risk for deferred preventive care for diabetes, and health systems might consider interventions to promote effective preventive services among chronically ill patients who frequently seek care for lower-priority conditions. Group visits among adults with chronic illness, for example, are associated with improved quality of care, clinical outcomes, and patient satisfaction, while reducing medical costs. ${ }^{23,24}$

The limitations of our study deserve consideration. First, based on diagnostic codes, our visit taxonomy may be prone to misclassification, and patients within each of the 3 use categories may have had different numbers of total visits. We believe, nevertheless, that our method of categorization provides a meaningful characterization of patients' longitudinal pattern of outpatient service use that may be useful to health planners. Second, we can only indirectly infer from diagnostic data that patient demand, rather than a clinician decision, stimulated the delivery of care for lowerpriority conditions. Investigators have consistently observed, however, that patient requests powerfully influence the content of primary care visits, ${ }^{20,21,25,26}$ and we believe it is unlikely that clinicians, in the absence of patient demand, would so commonly prioritize care for lower-priority conditions over diabetes care among a substantial fraction (12\%) of this population-based sample. Finally, we studied an insured population within a single health maintenance organization in the Pacific Northwest. The findings may not be generalizable to other populations with diabetes.

The strengths of our study include its large, population-based sample and our ability to control for a broad range of important covariates, including demographic and socioeconomic factors, diabetes complications, treatment intensity, chronic disease comorbidity, and depression status. Additionally, access to clinical and administrative databases for a closed population allowed us to create comprehensive accounts of patients' outpatient use during the study period, including both primary and specialty care, and to ascertain preventive service outcomes with a high degree of confidence. Enrolled in a prepaid health plan, patients had universal access to assigned primary care clinicians, minimizing confounding related to health care access.

We found that a pattern of infrequent use was a robust risk factor for failing to receive recommended preventive services for diabetes, and patients with frequent outpatient use for predominantly lower-priority diagnoses were at increased risk for deferred preventive services for diabetes. Although many of the health care needs of patients with chronic illness can be anticipated and planned, office practice remains organized principally to respond to patients' acute complaints. ${ }^{27}$ Innovations in the organization of primary care may be needed to ensure the delivery of evidence-based services in the context of sparse patient demand or demand for care for conditions that are relatively less amenable to effective medical intervention.

To read or post commentaries in response to this article, see it online at http://www.annfammed.org/cgi/content/full/4/1/32.

Key words: Chronic disease; disease management; preventive health services; diabetes; health services research; patient compliance; delivery of health care

Submitted May 12, 2005; submitted, revised, August 24, 2005; accepted September 15, 2005.

Funding support: This work was supported by National Institute of Mental Health grants MH-41739 and MH-01643 (Dr Wayne J. Katon, Principal Investigator) and American Cancer Society Mentored Research Scholar grant MRSGT-05-214-01-CPPB (to Dr Fenton). Dr Fenton was a Robert Wood Johnson Clinical Scholar during early phases of this project.

Disclaimer: The statements herein are the authors' and do not necessarily represent the views of the Robert Wood Johnson Foundation.

Acknowledgment: The authors thank Wayne Katon, Malia Oliver, Greg Simon, and other members of the Pathways study team for their assistance with this research.

\section{References}

1. Vijan S, Stevens DL, Herman WH, Funnell MM, Standiford CJ. Screening, prevention, counseling, and treatment for the complications of type II diabetes mellitus: putting evidence into practice. J Gen Intern Med. 1997; 12:567-580.

2. American Diabetes Association: clinical practice recommendations 2000. Diabetes Care. 2000;23(Suppl 1):S1-S116.

3. Beckles GL, Engelgau MM, Narayan KM, et al. Population-based assessment of the level of care among adults with diabetes in the U.S. Diabetes Care. 1998;21:1432-1438.

4. Streja DA, Rabkin SW. Factors associated with implementation of preventive care measures in patients with diabetes mellitus. Arch Intern Med. 1999;159:294-302.

5. Hippisley-Cox J, Pringle M. Prevalence, care, and outcomes for patients with diet-controlled diabetes in general practice: cross sectional survey. Lancet. 2004;364:423-428.

6. Redelmeier DA, Tan SH, Booth GL. The treatment of unrelated disorders in patients with chronic medical diseases. $N$ Engl J Med. 1998;338:1516-1520. 
7. Kiefe Cl, Funkhouser E, Fouad MN, May DS. Chronic disease as a barrier to breast and cervical cancer screening. J Gen Intern Med. 1998; 13:357-365.

8. Nutting PA, Baier M, Werner JJ, et al. Competing demands in the office visit: what influences mammography recommendations? J Am Board Fam Pract. 2001;14:352-361.

9. Yawn B, Goodwin MA, Zyzanski SJ, Stange KC. Time use during acute and chronic illness visits to a family physician. Fam Pract. 2003;20:474-477.

10. Katon W, von Korff M, Ciechanowski P, et al. Behavioral and clinical factors associated with depression among individuals with diabetes. Diabetes Care. 2004;27:914-920.

11. McCulloch DK, Price MJ, Hindmarsh M, Wagner EH. A populationbased approach to diabetes management in a primary care setting early results and lessons learned. Eff Clin Pract. 1998;1:12-22.

12. Oregon State Health Services Commission. Prioritized List of Health Services. Available at: http://egov.oregon.gov/DAS/OHPR/HSC/current_prior.shtml. Accessed August 12, 2005.

13. Bodenheimer T. The Oregon Health Plan-lessons for the nation. First of two parts. N Engl J Med. 1997;337:651-655.

14. Kroenke K, Spitzer RL, Williams JB. The PHQ-9: validity of a brief depression severity measure. J Gen Intern Med. 2001;16:606-613.

15. Rosenzweig JL, Weinger K, Poirier-Solomon L, Rushton M. Use of a disease severity index for evaluation of healthcare costs and management of comorbidities of patients with diabetes mellitus. Am J Manag Care. 2002;8:950-958.

16. Fishman PA, Goodman MJ, Hornbrook MC, et al. Risk adjustment using automated ambulatory pharmacy data: the RxRisk model. Med Care. 2003;41:84-99.

17. Rao RS, Sigurdson AJ, Doody MM, Graubard BI. An application of a weighting method to adjust for nonresponse in standardized incidence ratio analysis of cohort studies. Ann Epidemiol. 2005;15:129-136.
18. Harris MI. Health care and health status and outcomes for patients with type 2 diabetes. Diabetes Care. 2000;23:754-758.

19. Jaen CR, Stange KC, Nutting PA. Competing demands of primary care: a model for the delivery of clinical preventive services. J Fam Pract. 1994;38:166-171.

20. Chernof BA, Sherman SE, Lanto AB, et al. Health habit counseling amidst competing demands: effects of patient health habits and visit characteristics. Med Care. 1999;37:738-747.

21. Stange KC, Flocke SA, Goodwin MA, Kelly RB, Zyzanski SJ. Direct observation of rates of preventive service delivery in community family practice. Prev Med. 2000;31:167-176.

22. Nutting PA, Rost K, Smith J, Werner JJ, Elliot C. Competing demands from physical problems: effect on initiating and completing depression care over 6 months. Arch Fam Med. 2000;9:1059-1064.

23. Beck A, Scott J, Williams $P$, et al. A randomized trial of group outpatient visits for chronically ill older HMO members: the Cooperative Health Care Clinic. J Am Geriatr Soc. 1997;45:543-549.

24. Wagner EH, Grothaus LC, Sandhu N, et al. Chronic care clinics for diabetes in primary care: a system-wide randomized trial. Diabetes Care. 2001;24:695-700.

25. Kravitz RL, Bell RA, Azari R, et al. Direct observation of requests for clinical services in office practice: what do patients want and do they get it? Arch Intern Med. 2003;163:1673-1681

26. Kravitz RL, Epstein RM, Feldman MD, et al. Influence of patients requests for direct-to-consumer advertised antidepressants: a randomized controlled trial. JAMA. 2005;293:1995-2002.

27. Wagner EH, Austin BT, Von Korff M. Organizing care for patients with chronic illness. Milbank Q. 1996;74:511-544. 